\title{
Singlet Fission in Quaterrylenediimide Thin Films
}

\author{
Michelle Chen, Natalia E. Powers-Riggs, Adam F. Coleman, Ryan M. Young*, and \\ Michael R. Wasielewski* \\ Department of Chemistry and Institute for Sustainability and Energy at Northwestern, \\ Northwestern University, Evanston IL 60208-3113
}

1. Crystallographic Data S2-3

a. Torsion Angles and Molecular Packing Distances ..................................................... S2

b. Crystal Structure Parameters for ArQDI ...................................................................... S3

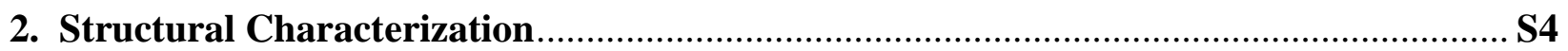

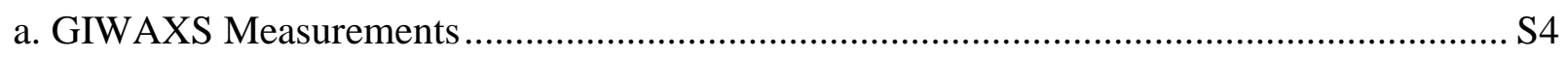

b. GIXD Out-of-Plane Diffractograms ........................................................................ S4

3. Data Processing and Global Analysis Description ...............................................S5-S6

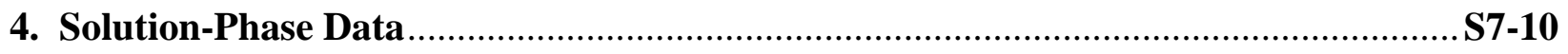

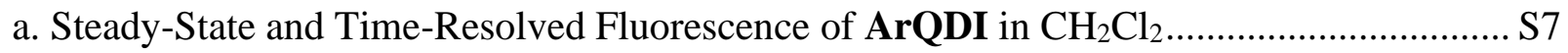

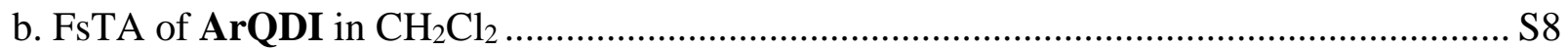

c. Chemically Reduced and Oxidized ArQDI Spectra .................................................. S9

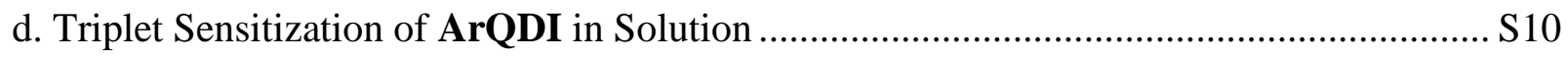

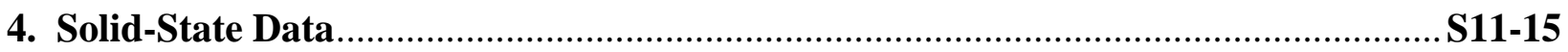

a. Global Fit of the $100 \mathrm{kHz}$ fsTA Data of the $\mathrm{CH}_{2} \mathrm{Cl}_{2}$ Solvent-Vapor Annealed Film ........S11

b. Global Fit of the $1 \mathrm{kHz}$ fsTA Data of the $\mathrm{CH}_{2} \mathrm{Cl}_{2}$ Solvent-Vapor Annealed Film ........... S12

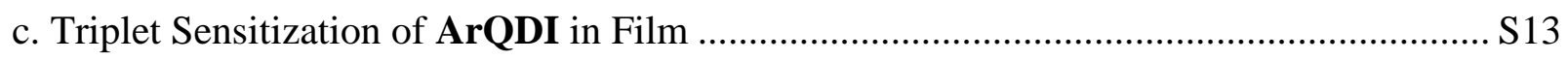

d. Global Fit of the nsTA Data of the $\mathrm{CH}_{2} \mathrm{Cl}_{2}$ Solvent-Vapor Annealed Film .................... S14

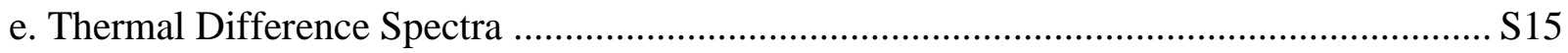

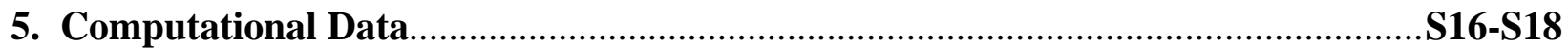

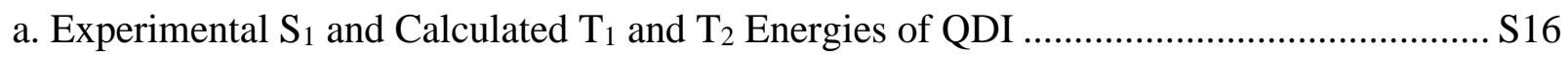

b. Full $N, N$-dimethyl QDI Geometries ............................................................... S16-18

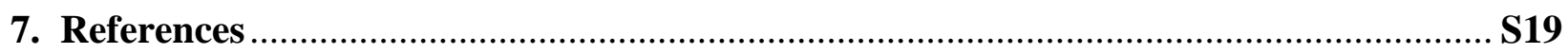




\section{Crystallographic Data}

\section{Torsion Angles and Molecular Packing Distances}

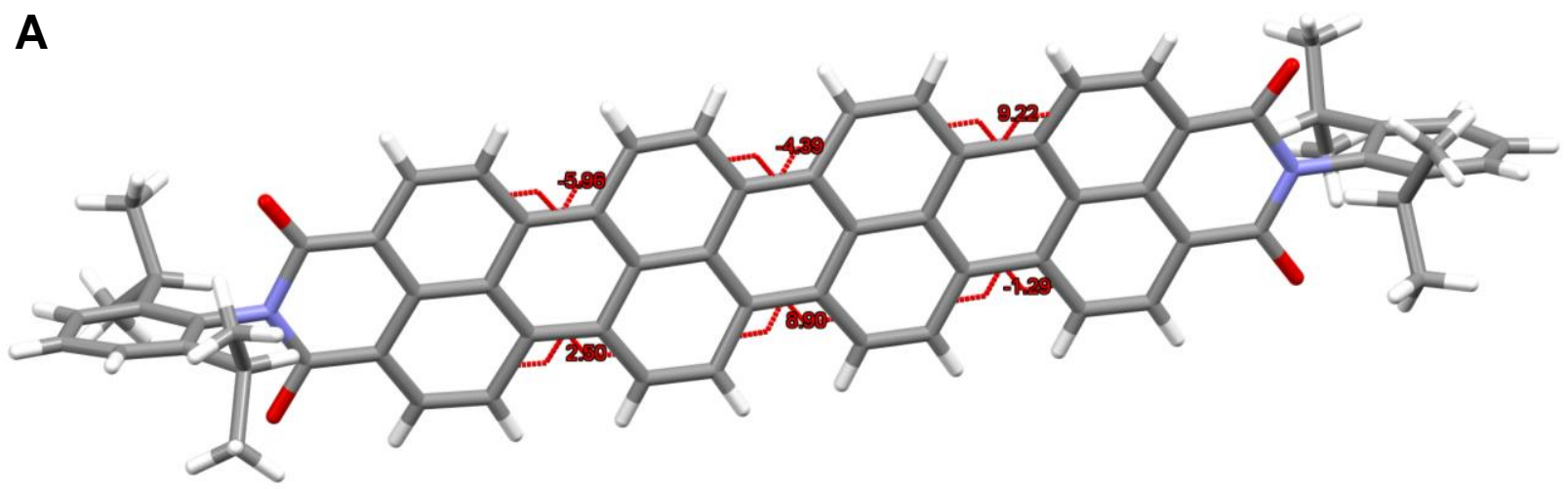

B

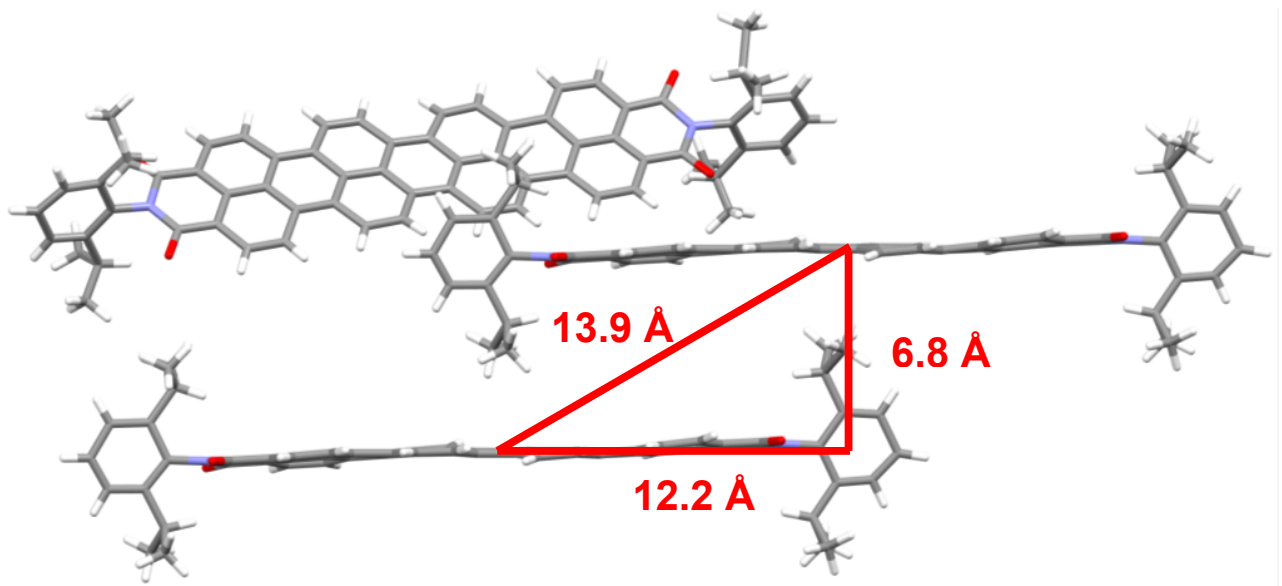

C

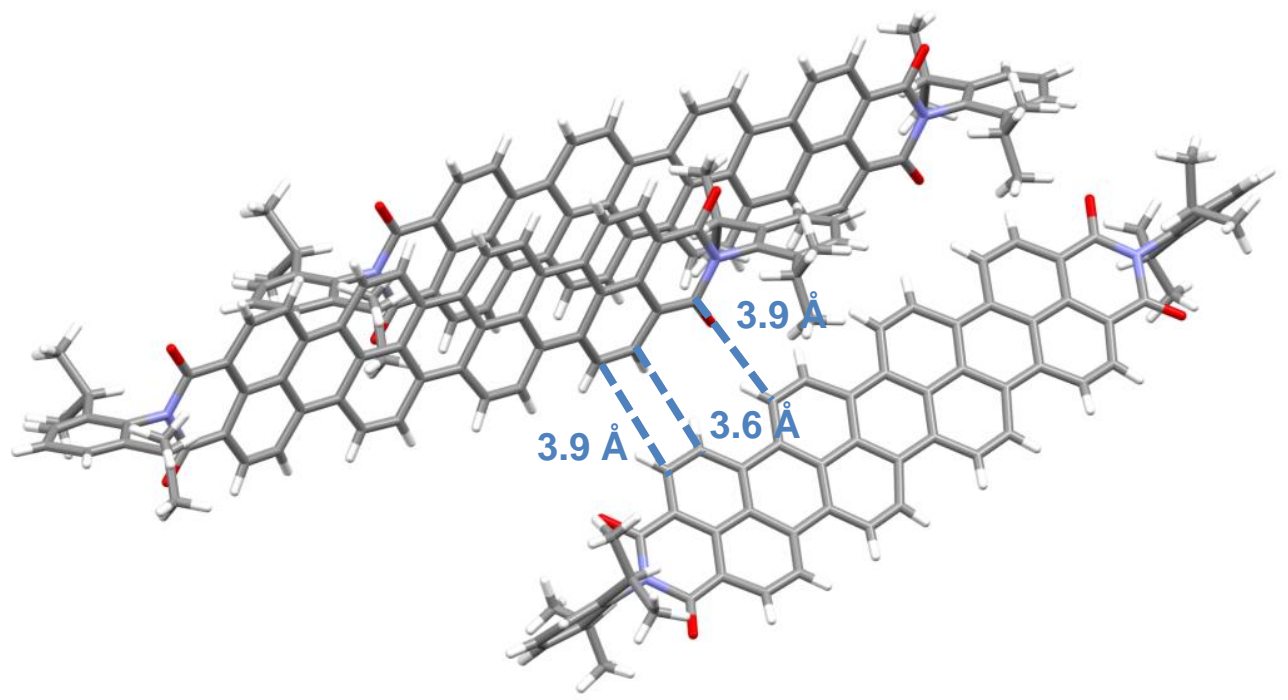

Figure S1. (A) ArQDI crystal structure with selected torsion angles. Molecular packing distances for the (B) ArQDI $\pi$-core stacked and (C) edge-on geometries. 


\section{Crystal Structure Parameters for ArQDI}

Table S1. Crystal Data and Structure Refinement for ArQDI

Identification code

Empirical Formula

Formula Weight

Temperature/K

Crystal System

Space Group

$\mathrm{a} / \AA$

$\mathrm{b} / \AA$

$\mathrm{c} / \AA$

$\alpha /{ }^{\circ}$

$\beta /{ }^{\circ}$

$\gamma / 0$

Volume $/ \AA^{3}$

$\mathrm{Z}$

$\rho_{\text {cald }} / \mathrm{g} / \mathrm{cm}^{3}$

$\mu / \mathrm{mm}^{-1}$

$\mathrm{F}(000)$

Crystal Size $/ \mathrm{mm}^{3}$

Radiation

$2 \Theta$ range for data collection $/{ }^{\circ}$

Index Ranges

Reflections Collected

Independent Reflections

Data/Restraints/Parameters

Goodness-of-fit on $\mathrm{F}^{2}$

Final R Indexes [I $>2 \sigma(\mathrm{I})]$

Final R Indexes [all data]

Largest diff. peak/hole / e $\AA^{-1}$

Flack Parameter
(iPr2Ph)2QDI

$\mathrm{C}_{68} \mathrm{H}_{50} \mathrm{~N}_{2} \mathrm{O}_{4}$

959.16

99.99

monoclinic

$\mathrm{P} 21$

13.949(3)

15.719(3)

$15.272(3)$

90

$107.438(13)$

90

3194.9(10)

2

0.997

0.483

1008.0

$0.166 \times 0.058 \times 0.057$

$\operatorname{CuK} \alpha(\lambda=1.54178)$

8.274 to 117.846

$-8 \leq \mathrm{h} \leq 15,-17 \leq \mathrm{k} \leq 17,-16 \leq 1 \leq 16$

9798

$6414\left[\mathrm{R}_{\text {int }}=0.0424, \mathrm{R}_{\text {sigma }}=0.1498\right]$

$6414 / 37 / 675$

1.052

$\mathrm{R}_{1}=0.1205, \mathrm{w} \mathrm{R}_{2}=0.3002$

$\mathrm{R}_{1}=0.1661, \mathrm{wR}_{2}=0.3549$

$0.64 /-0.36$

$0.3(8)$ 


\section{Structural Characterization}

\section{GIWAXS Measurements}

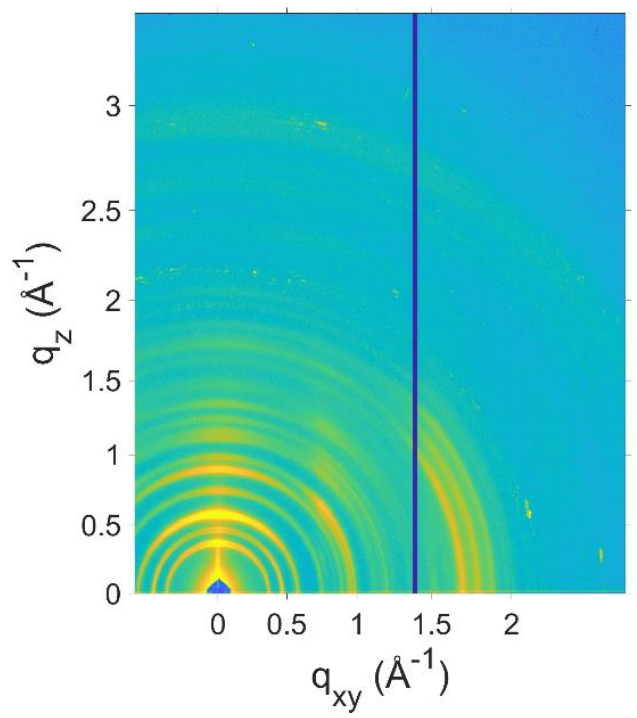

Figure S2. GIWAXS spectra of the $\mathrm{CH}_{2} \mathrm{Cl}_{2}$ solvent-vapor annealed (SVA) film.

\section{GIXD Out-of-Plane Diffractograms}

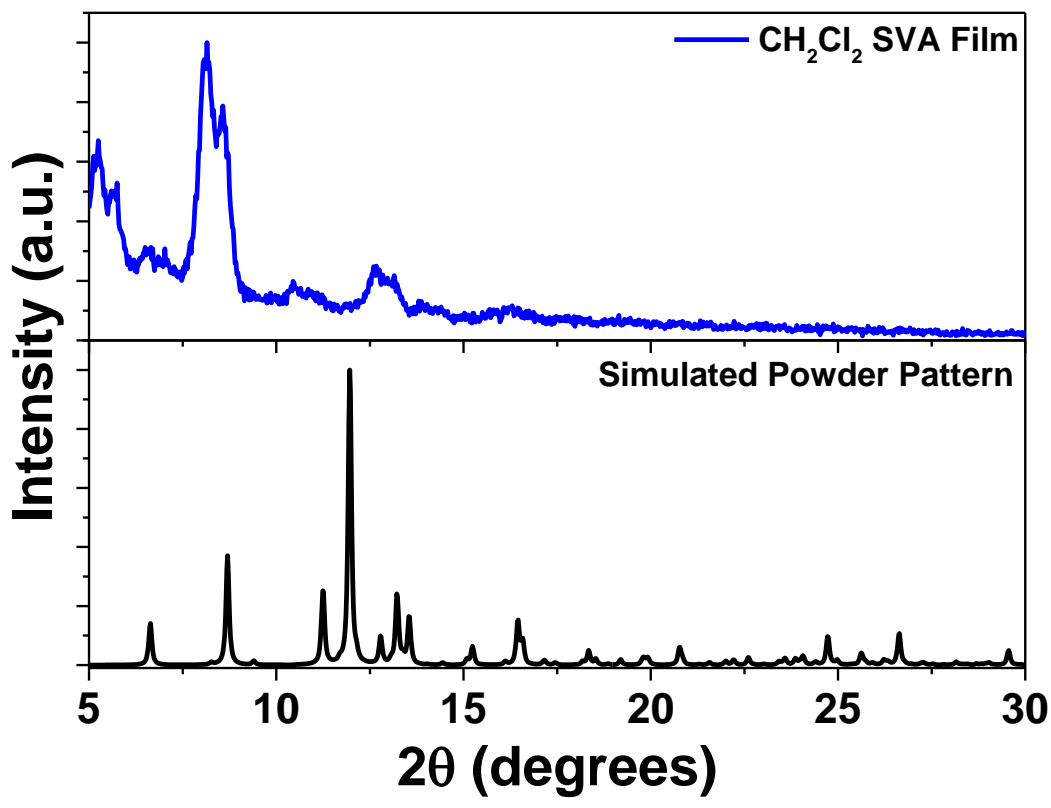

Figure S3. GIXD diffractogram (out-of-plane) of the $\mathrm{CH}_{2} \mathrm{Cl}_{2}$ solvent-vapor annealed (SVA) (blue) film compared to the simulated powder pattern (black). 


\section{Data Processing and Global Analysis Description}

Femtosecond Transient Absorption Spectroscopy (100 kHz): The $100 \mathrm{kHz}$ fsTA data are scattersubtracted and chirp-corrected using Surface Xplorer 4 (Ultrafast Systems, LLC) prior to kinetic analysis. The data analysis was performed with home written programs in MATLAB and based on a global fit to selected single-wavelength kinetics. A uniform instrument response of $w=300$ fs (full width at half maximum, FWHM) across the frequency domain was assumed. Additionally, a shared time-zero $\left(t_{0}\right)$ is implicit in the global analysis. Details of the global analysis have been described previously. ${ }^{1}$ The first-order kinetic model with rate matrix $K$ used to model the ArQDI film is given below:

1) ArQDI in Film:

$$
\underline{\underline{K}}=\left[\begin{array}{cccc}
-k_{A \rightarrow B} & 0 & 0 & 0 \\
k_{A \rightarrow B} & -k_{B \rightarrow C} & 0 & 0 \\
0 & k_{B \rightarrow C} & -k_{C \rightarrow D} & 0 \\
0 & 0 & k_{C \rightarrow D} & -k_{D \rightarrow G S}
\end{array}\right] \text { (Eqn. S1) }
$$

Femtosecond Transient Absorption Spectroscopy (1 kHz): In addition to scatter-subtraction and chirp-correction, the visible and NIR data sets collected on the $1 \mathrm{kHz}$ instrument are spectrally merged using Surface Xplorer 4 (Ultrafast Systems, LLC). The $1 \mathrm{kHz}$ data was fit using a similar procedure as the fsTA data but considers singlet-singlet annihilation in the solid-state. The firstorder kinetic models with rate matrix $K$ and higher-order kinetic models of each system are given below:

1) ArQDI in $\mathrm{CH}_{2} \mathrm{Cl}_{2}$ :

$$
\underline{\underline{K}}=\left[\begin{array}{cc}
-k_{A \rightarrow B} & 0 \\
k_{A \rightarrow B} & -k_{B \rightarrow G S}
\end{array}\right]
$$




\section{2) ArQDI in Film:}

$$
\begin{aligned}
& \frac{d[A]}{d t}=\frac{-k_{S S A}}{2}[A]^{2}-k_{A \rightarrow B}[A] \\
& \frac{d[B]}{d t}=k_{A \rightarrow B}[A]-k_{B \rightarrow C}[B] \\
& \frac{d[C]}{d t}=k_{B \rightarrow C}[B]-k_{C \rightarrow D}[C] \\
& \frac{d[D]}{d t}=k_{C \rightarrow D}[C]-k_{D \rightarrow G S}[D]
\end{aligned}
$$

Nanosecond Transient Absorption Spectroscopy: The nsTA data are processed and fit using a similar procedure as the $100 \mathrm{kHz}$ fsTA data. A uniform instrument response of $w=600$ ps was used instead of $w=300 \mathrm{fs}$ in the global analysis. Below is the first-order kinetic model with rate matrix $K$ for ArQDI in film:

1) ArQDI in Film:

$$
\underline{\underline{K}}=\left[\begin{array}{cc}
-k_{A \rightarrow B} & 0 \\
k_{A \rightarrow B} & -k_{B \rightarrow G S}
\end{array}\right]
$$




\section{Solution-Phase Data}

Steady-State and Time-Resolved Fluorescence of ArQDI in $\mathrm{CH}_{2} \mathrm{Cl}_{2}$

A
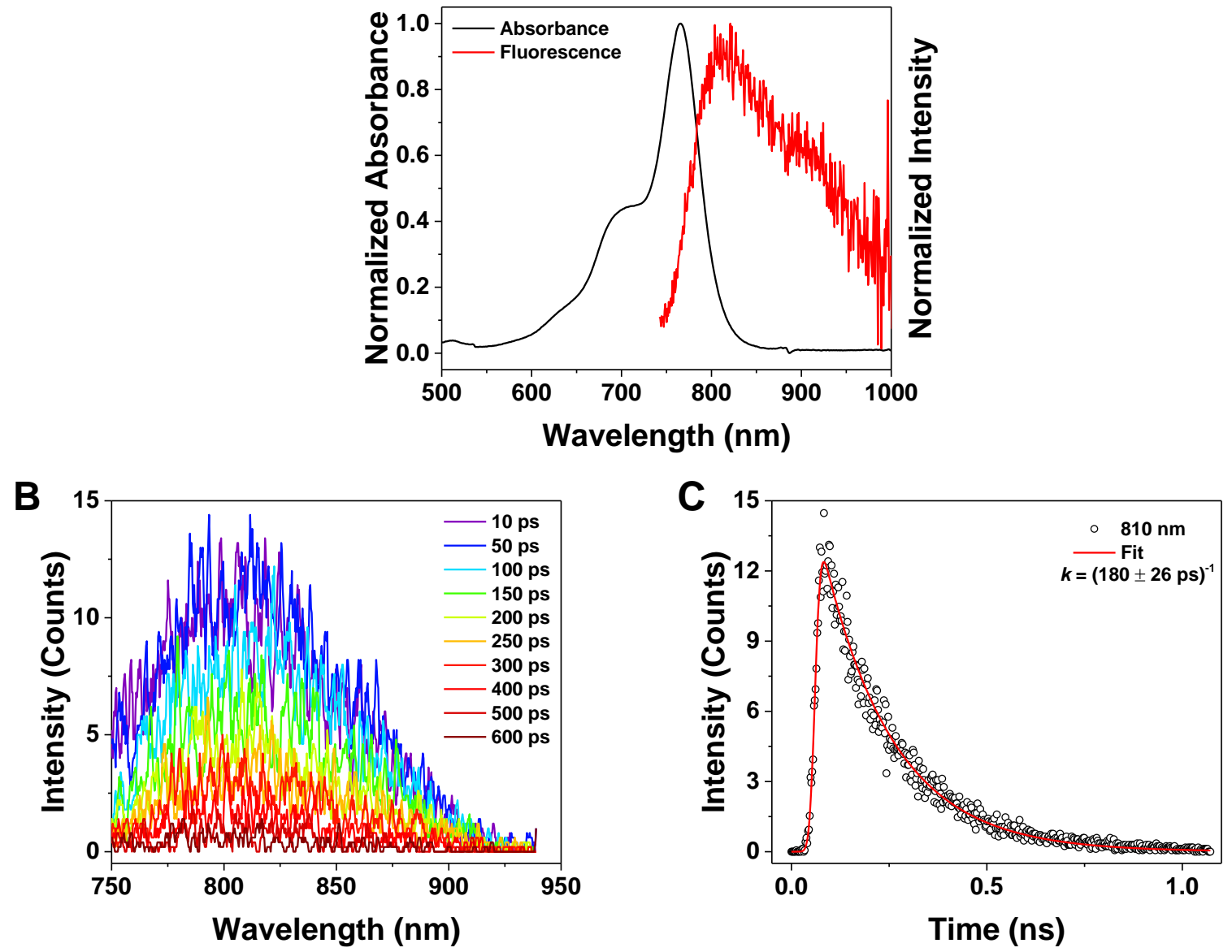

Figure S4. (A) Steady-state absorption (black) and fluorescence (red) spectra of ArQDI in $\mathrm{CH}_{2} \mathrm{Cl}_{2}$. (B) TRF spectra at selected time delays of ArQDI in $\mathrm{CH}_{2} \mathrm{Cl}_{2}$ collected with a $1 \mathrm{~ns}$ window; the spectra were smoothed with 5-point adjacent averaging. Data were collected with $\lambda_{\mathrm{ex}}=695 \mathrm{~nm}$. (C) Single-wavelength kinetic fits at $810 \mathrm{~nm}$. 


\section{FsTA of ArQDI in $\mathrm{CH}_{2} \mathrm{Cl}_{2}$}

A

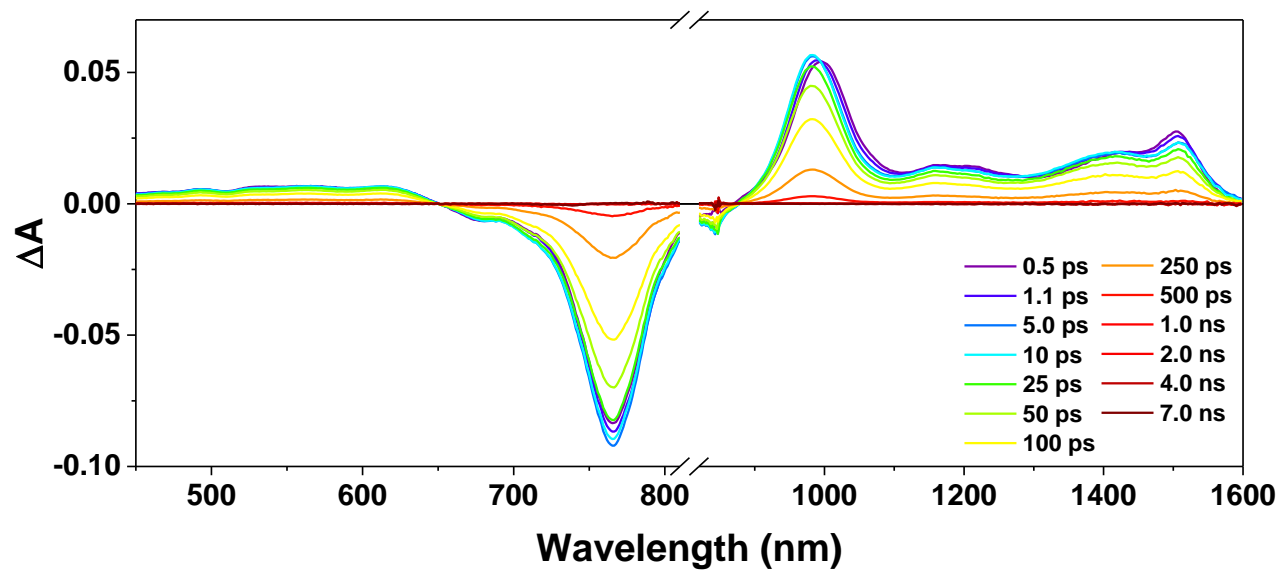

B
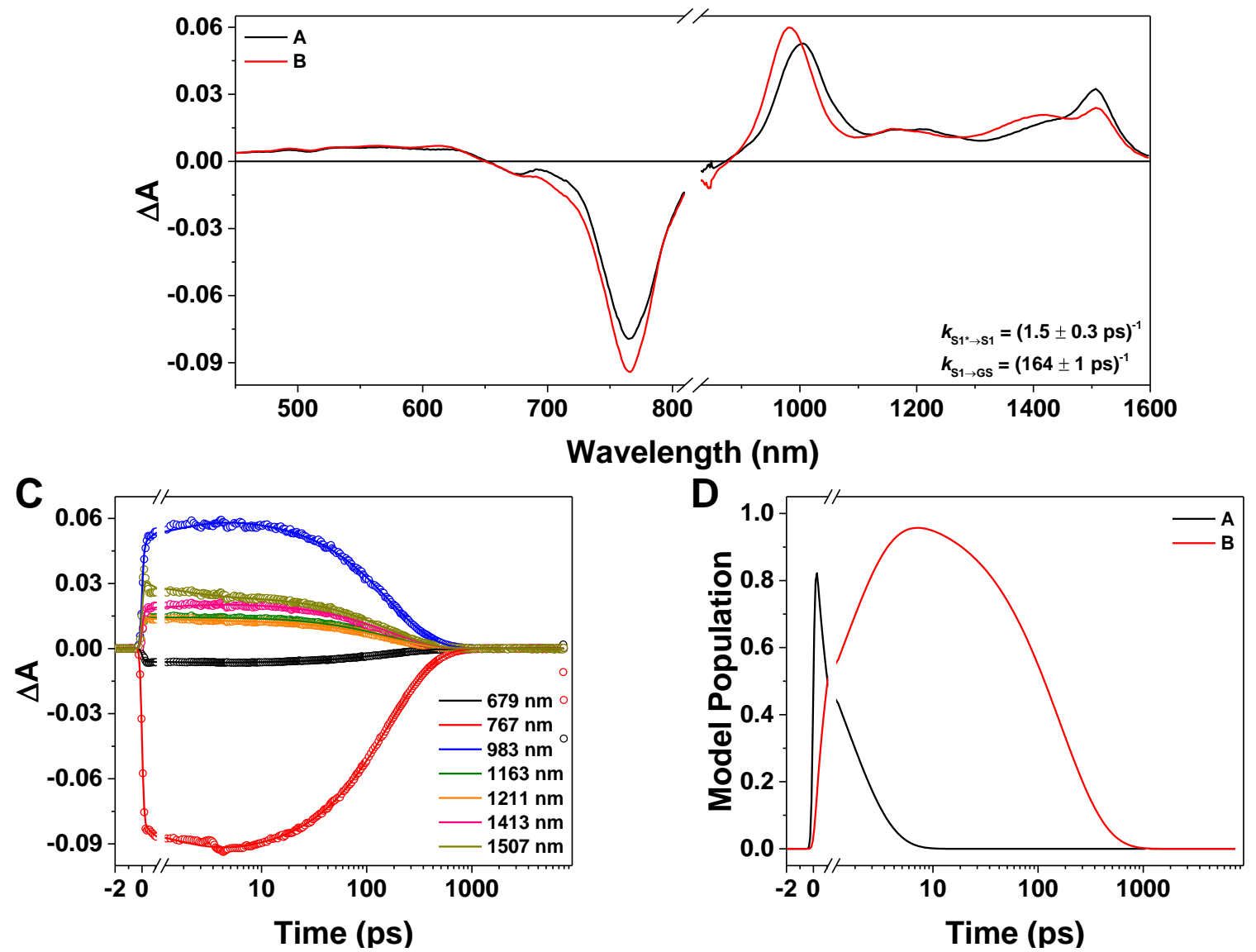

Figure S5. (A) FsTA spectra at selected time delays, (B) evolution-associated spectra, (C) single-wavelength kinetics at 679 (black), 767 (red), 983 (blue), 1163 (green), 1211 (orange), 1413 (pink), $1507 \mathrm{~nm}$ (dark yellow), and (D) population dynamics obtained by global analysis with an $\mathrm{A} \rightarrow \mathrm{B} \rightarrow$ ground model of ArQDI in $\mathrm{CH}_{2} \mathrm{Cl}_{2}, \lambda_{\mathrm{ex}}=740 \mathrm{~nm}$. 
Chemically Reduced and Oxidized ArQDI Spectra

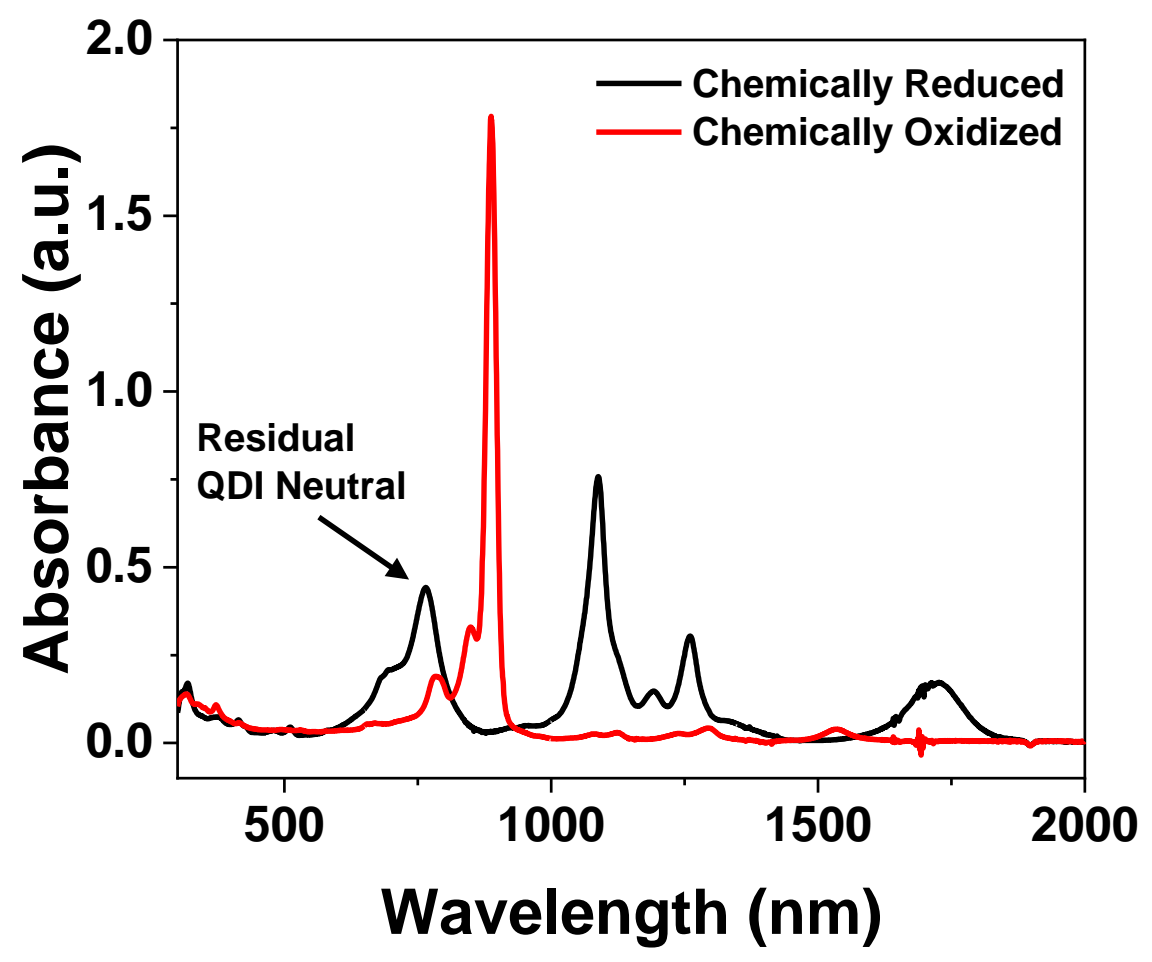

Figure S6. Partially chemically reduced (black) and oxidized (red) spectra of ArQDI in $\mathrm{CH}_{2} \mathrm{Cl}_{2}$ in the presence of cobaltocene and $\mathrm{NOBF}_{4}$, respectively. 


\section{Triplet Sensitization of ArQDI in Solution}

Triplet sensitization of ArQDI was performed in toluene by photoexcitation of anthracene, in large excess, at $355 \mathrm{~nm}$. Rapid intersystem crossing populates the anthracene $\mathrm{T}_{1}$ state $(1.85 \mathrm{eV})$; the anthracene $\mathrm{T}_{1}$ state triplet energy transfers to the lower energy QDI $\mathrm{T}_{1}$, which allows for optical characterization of the QDI $\mathrm{T}_{1}$ state by nsTA. The sample was deoxygenated by three freeze-pumpthaw cycles.

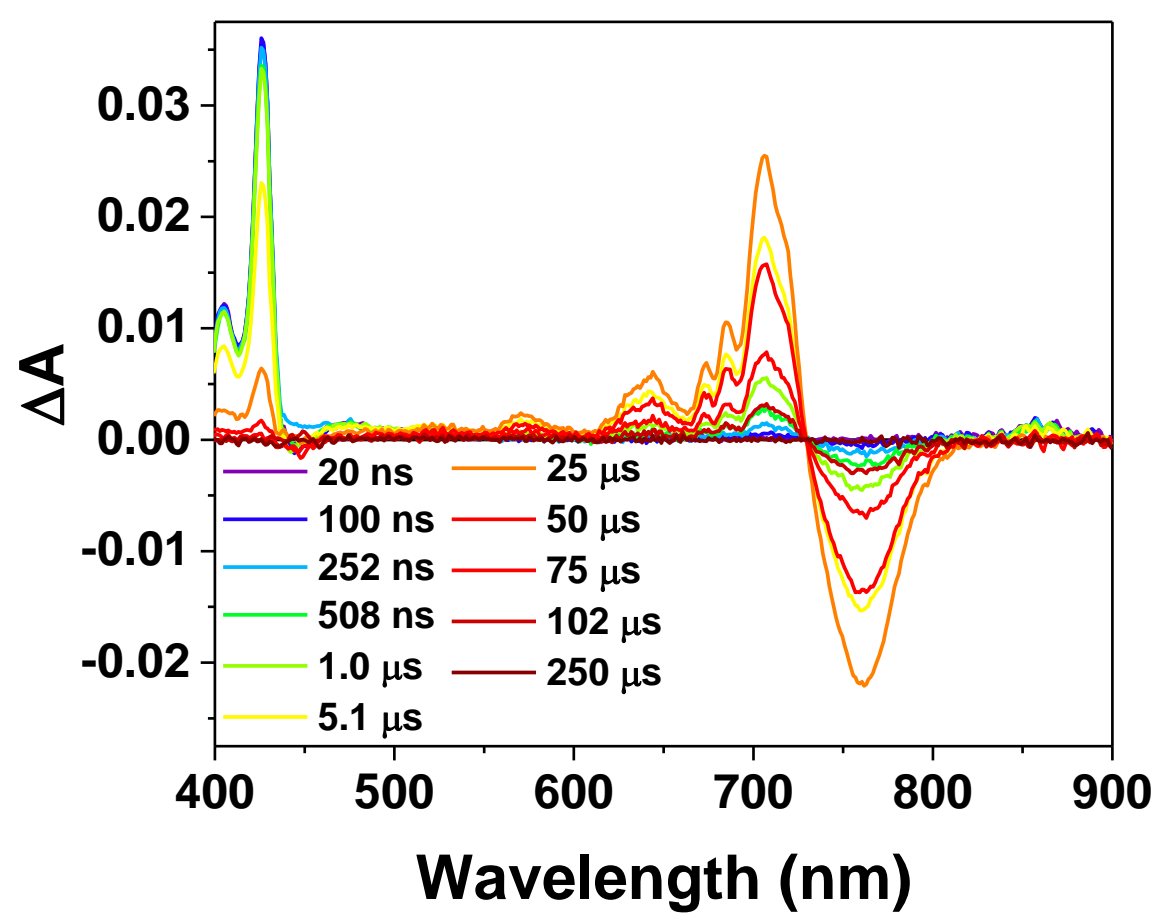

Figure S7. NsTA spectra of anthracene-sensitized ArQDI (orange to red, $\Delta \mathrm{t}>25$ $\mu \mathrm{s})$ in toluene, $\lambda_{\mathrm{ex}}=355 \mathrm{~nm}$. 


\section{Global Fit of the $100 \mathrm{kHz}$ fsTA Data of the $\mathrm{CH}_{2} \mathrm{Cl}_{2}$ Solvent-Vapor Annealed Film}

A

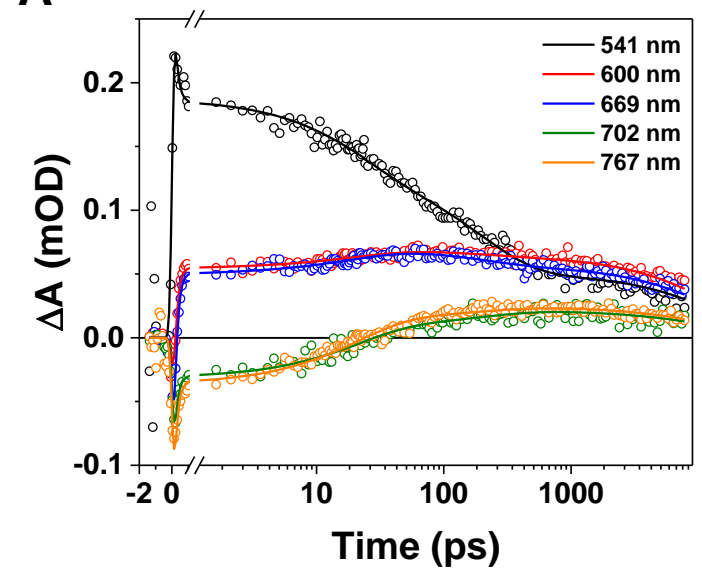

B

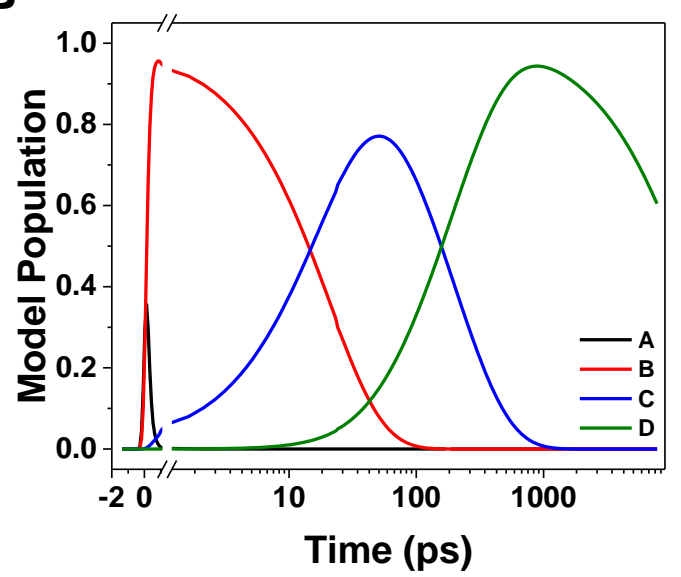

Figure S8. (A) Single-wavelength kinetics at 541 (black), 600 (red), 669 (blue), 702 (green), and 767 (orange) and (C) population dynamics obtained by global analysis with an $\mathrm{A} \rightarrow \mathrm{B} \rightarrow$ $\mathrm{C} \rightarrow \mathrm{D} \rightarrow$ ground model of the $\mathrm{CH}_{2} \mathrm{Cl}_{2}$ solvent-vapor annealed ArQDI film, $\lambda_{\mathrm{ex}}=740 \mathrm{~nm}$. 


\section{Global Fit of the $1 \mathrm{kHz}$ fsTA Data of the $\mathrm{CH}_{2} \mathrm{Cl}_{2}$ Solvent-Vapor Annealed Film}

A

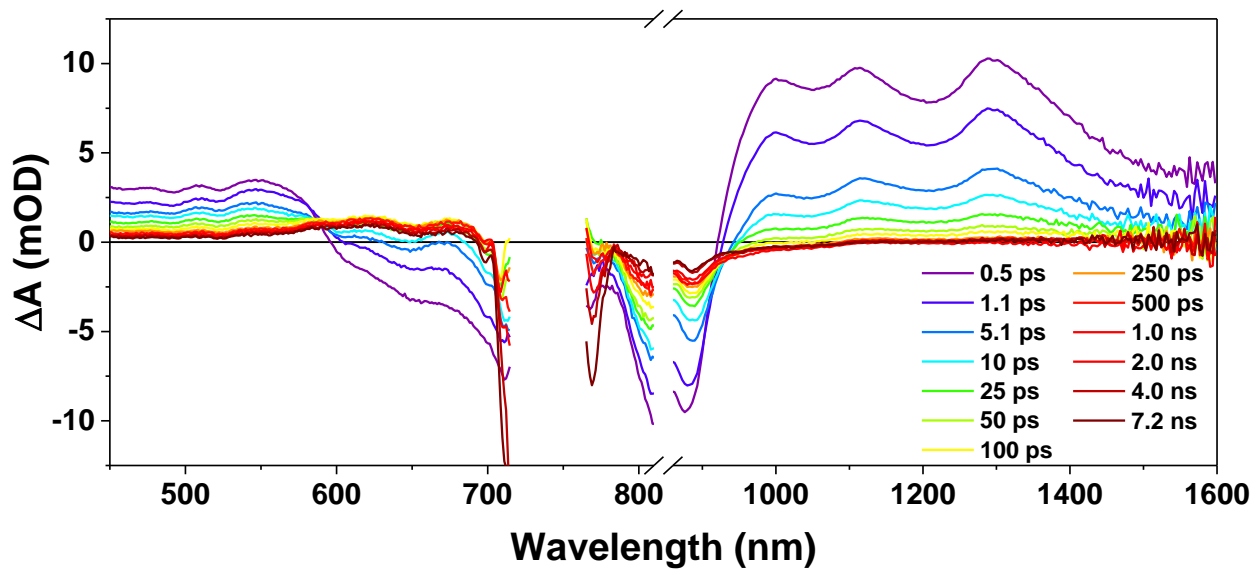

B

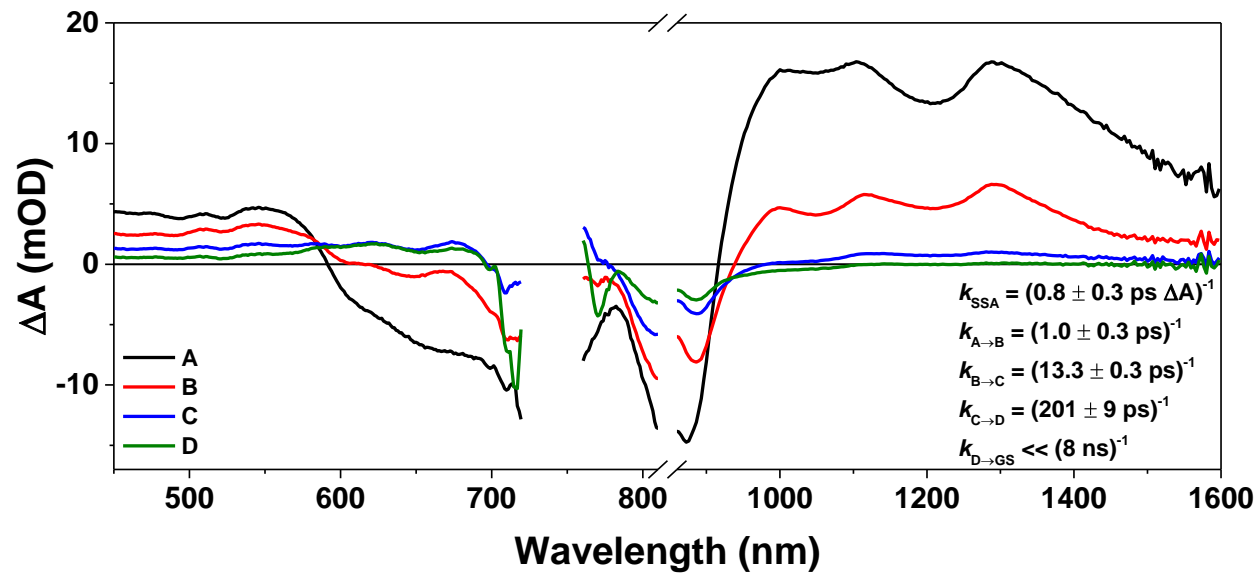

C

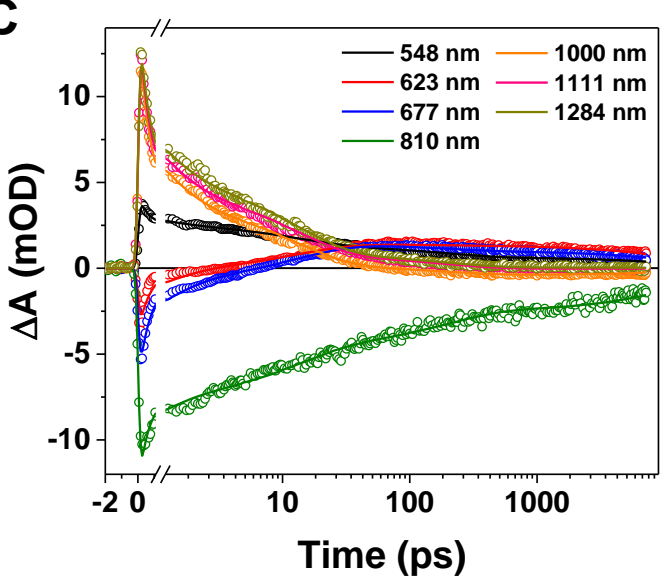

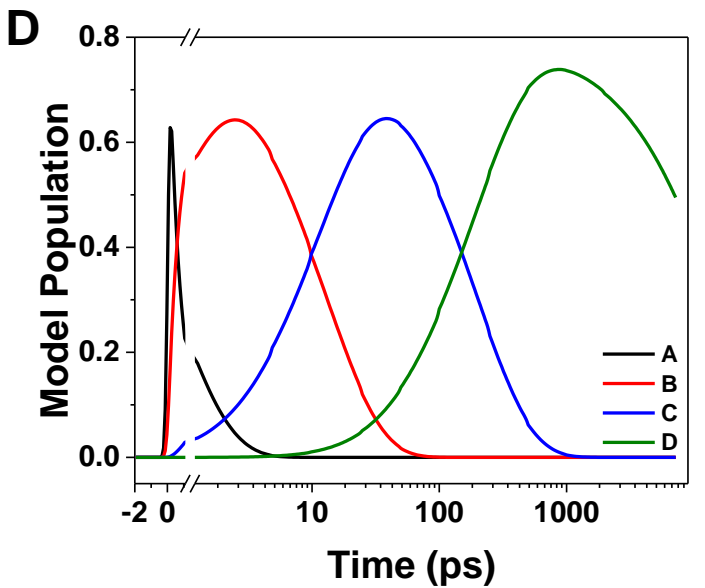

Figure S9. (A) FsTA spectra at selected time delays, (B) evolution-associated spectra, (C) single-wavelength kinetics at 548 (black), 623 (red), 677 (blue), 810 (green), 1000 (orange), 1111 (pink), $1284 \mathrm{~nm}$ (dark yellow), and (D) population dynamics obtained by global analysis with an $\mathrm{A} \rightarrow \mathrm{B} \rightarrow \mathrm{C} \rightarrow \mathrm{D} \rightarrow$ ground model, which incorporates singlet-singlet annihilation, of the $\mathrm{CH}_{2} \mathrm{Cl}_{2}$ solvent-vapor annealed ArQDI film, $\lambda_{\text {ex }}=740 \mathrm{~nm}$. 


\section{Triplet Sensitization of ArQDI in Film}

Triplet sensitization of ArQDI in film was performed by photoexciting an $\mathbf{A r Q D I} / \mathrm{Pd}_{(}\left(\mathrm{PPh}_{3}\right)_{2} \mathrm{Cl}_{2}$ $(10: 1 \mathrm{w} / \mathrm{w})$ doped film at $740 \mathrm{~nm}$ where $\mathrm{Pd}\left(\mathrm{PPh}_{3}\right)_{2} \mathrm{Cl}_{2}$ is the heavy-atom source used to induce spin-orbit intersystem crossing. Photoexcitation results in population of the ArQDI singlet excited state, which then undergoes ultrafast SF and spin-orbit intersystem crossing. The resulting longtime spectra are of the $\left(\mathrm{T}_{1} \mathrm{~T}_{1}\right)$ state and triplet excitons formed by both processes.

A

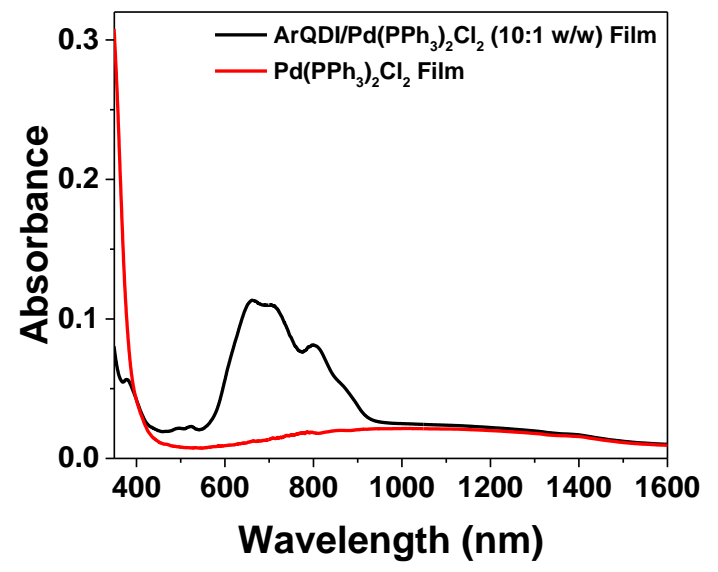

B

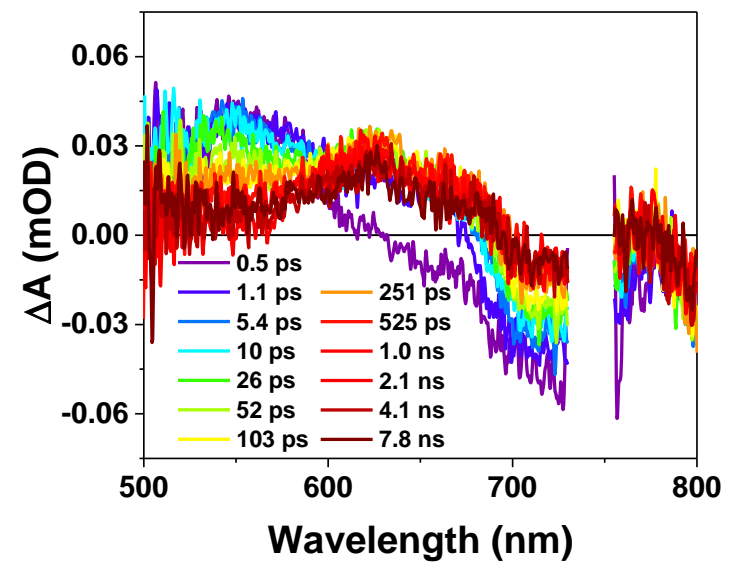

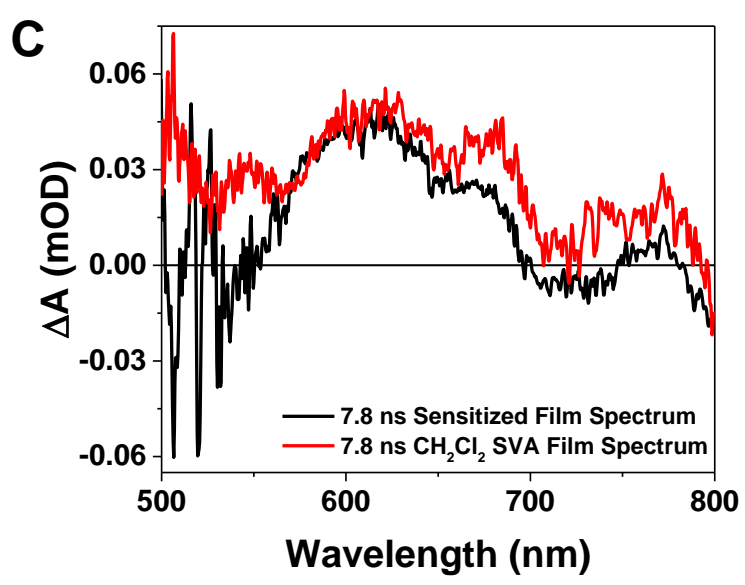

Figure S10. (A) Scatter-corrected absorbance spectra of the thermally annealed $\left(120{ }^{\circ} \mathrm{C}, 2 \mathrm{~h}\right)$ ArQDI/Pd( $\left(\mathrm{PPh}_{3}\right)_{2} \mathrm{Cl}_{2}$ and $\mathrm{Pd}\left(\mathrm{PPh}_{3}\right)_{2} \mathrm{Cl}_{2}$ films. (B) FsTA spectra at selected time delays of the thermally annealed ArQDI/Pd( $\left(\mathrm{PPh}_{3}\right)_{2} \mathrm{Cl}_{2}$ film, $\lambda_{\mathrm{ex}}=740 \mathrm{~nm}$. (C) Overlay of the sensitized triplet spectrum at $7.8 \mathrm{~ns}$ (black) with the $7.8 \mathrm{~ns}$ fsTA spectrum of the $\mathrm{CH}_{2} \mathrm{Cl}_{2} \mathrm{SVA}$ film (red). 


\section{Global Fit of the nsTA Data of the $\mathrm{CH}_{2} \mathrm{Cl}_{2}$ Solvent-Vapor Annealed Film}

A

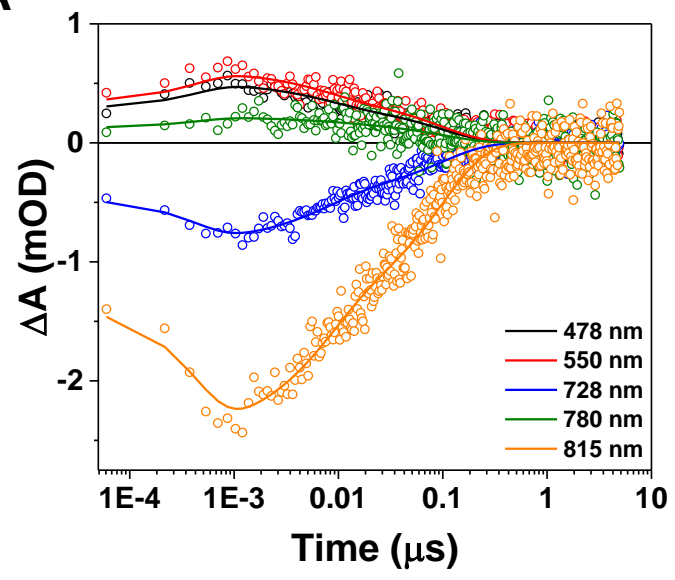

B

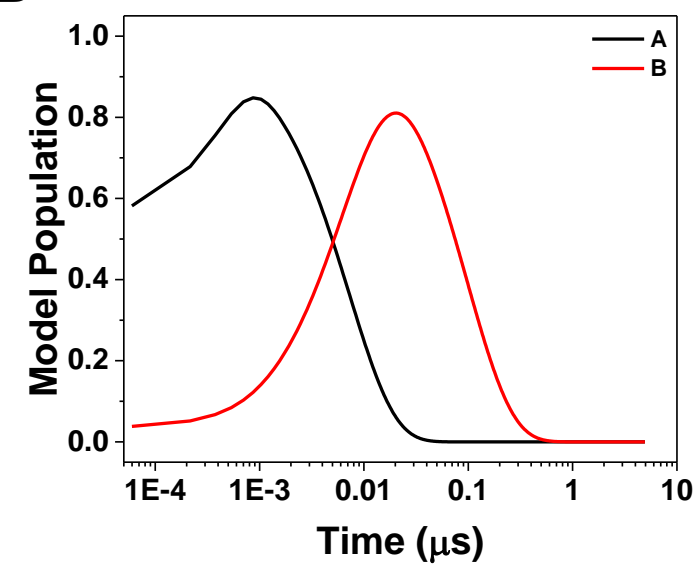

Figure S11. (A) single-wavelength kinetics at 478 (black), 550 (red), 728 (blue), 780 (green), and $815 \mathrm{~nm}$ (orange) and (D) population dynamics obtained by global analysis with an $\mathrm{A} \rightarrow \mathrm{B}$ ground model of the $\mathrm{CH}_{2} \mathrm{Cl}_{2}$ solvent vapor-annealed ArQDI film, $\lambda_{\mathrm{ex}}=600 \mathrm{~nm}$. 


\section{Thermal Difference Spectra}

A
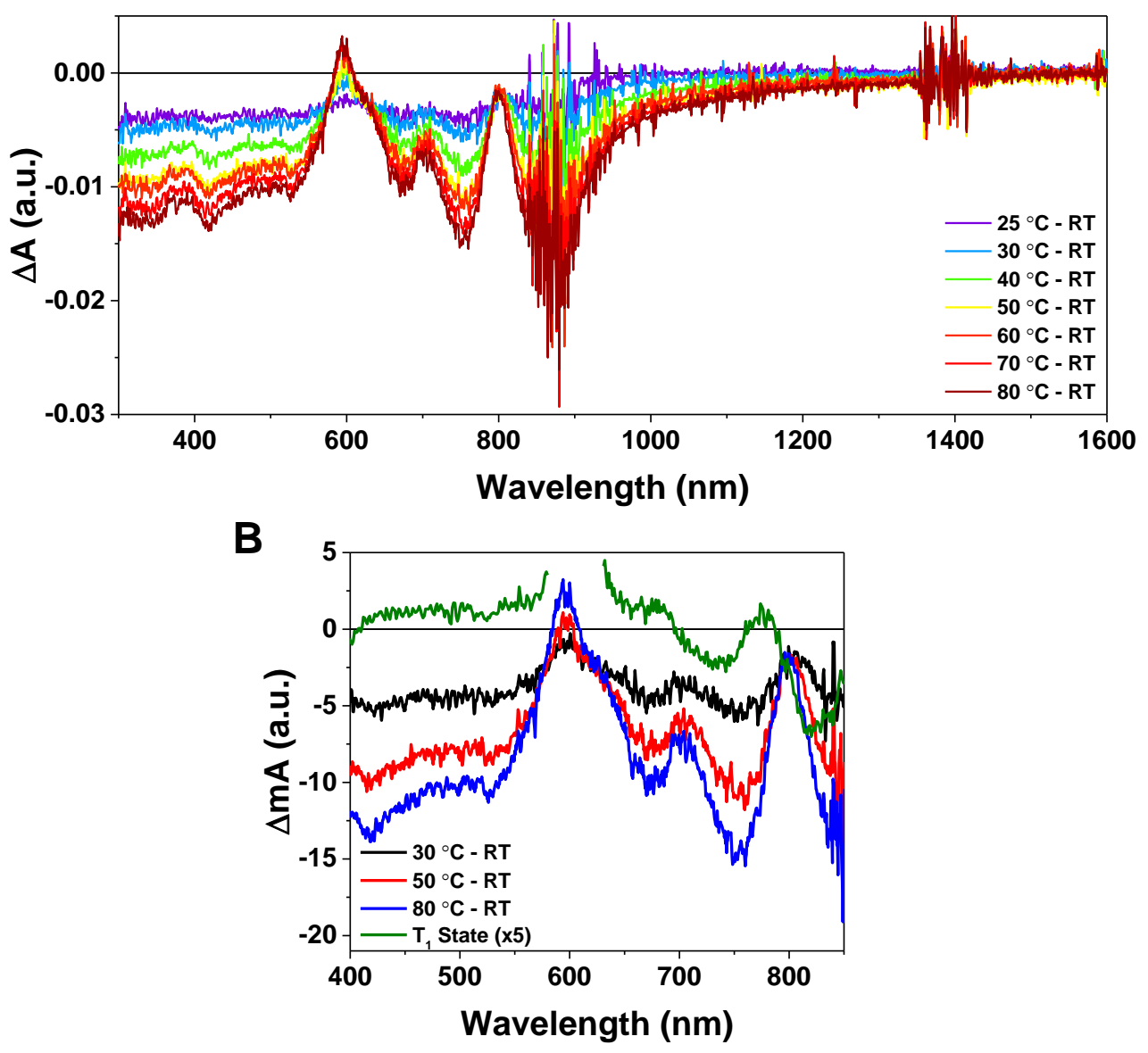

Figure S12. (A) Thermal difference spectra of the $\mathrm{CH}_{2} \mathrm{Cl}_{2} \mathrm{SVA}$ ArQDI film. (B) Comparisons of the thermal difference spectra and the $\mathrm{T}_{1}$ state. 


\section{Computational Data}

Table S2. Experimental $\mathrm{S}_{1}$ and Calculated $\mathrm{T}_{1}$ and $\mathrm{T}_{2}$ Energies of QDI

\begin{tabular}{|c|c|c|c|c|c|}
\hline Compound & $\mathbf{S}_{1}(\mathbf{e V})$ Exp. $^{\mathbf{a}}$ & $\begin{array}{c}\mathbf{T}_{\mathbf{1}}(\mathbf{e V}) \\
\text { Calcd. } \\
\text { UB3LYP }\end{array}$ & $\begin{array}{c}\mathbf{T}_{\mathbf{1}}(\mathbf{e V}) \\
\text { Calcd. }_{\text {TDDFT }}\end{array}$ & $\begin{array}{c}\mathbf{T}_{\mathbf{2}}(\mathbf{e V}) \\
\text { Calcd. } \\
\text { TDDFT } \\
\text { from } \mathbf{S}_{0} \\
\text { geometry }^{\mathbf{b}}\end{array}$ & $\begin{array}{c}\mathbf{T}_{\mathbf{2}}(\mathbf{e V}) \\
\text { Calcd. } \\
\text { TDDFT } \\
\text { from T1 } \\
\text { geometry }^{\mathbf{c}}\end{array}$ \\
\hline $\begin{array}{c}N, N \text {-dimethyl } \\
\text { QDI }\end{array}$ & 1.60 & 0.64 & 0.62 & 1.56 & 1.73 \\
\hline
\end{tabular}

${ }^{a}$ Experimental $\mathrm{S}_{1}$ energy is determined from the optical band gap of ArQDI in solution.

${ }^{\mathrm{b}}$ TDDFT calculation was performed on the optimized ground state singlet geometry.

${ }^{c}$ TDDFT calculation was performed on the optimized UB3LYP $\mathrm{T}_{1}$ geometry.

$N, N$-dimethyl QDI: Singlet Ground State

$\begin{array}{lrrrr}\mathrm{C} & 7.2114232775 & 2.4207929681 & -0.0062903066 & \mathrm{C} \\ \mathrm{C} & 5.8148793660 & 2.4298812537 & -0.0095066601 & \mathrm{C} \\ \mathrm{C} & 5.0671503738 & 1.2462699816 & -0.0057215648 & \mathrm{C} \\ \mathrm{C} & 5.7679652587 & -0.0002562867 & 0.0013016918 & \mathrm{C} \\ \mathrm{C} & 7.1976062247 & -0.0008093688 & 0.0051419331 & \mathrm{C} \\ \mathrm{C} & 7.9088556977 & 1.2229951549 & 0.0011736882 & \mathrm{C} \\ \mathrm{C} & 5.0663825958 & -1.2461225323 & 0.0048400351 & \mathrm{~N} \\ \mathrm{C} & 5.8133408309 & -2.4301028083 & 0.0129000030 & \mathrm{C} \\ \mathrm{C} & 7.2096076439 & -2.4218805499 & 0.0168531354 & \mathrm{C} \\ \mathrm{C} & 7.9089447309 & -1.2248436227 & 0.0128933914 & \mathrm{~N} \\ \mathrm{C} & 3.6016135257 & 1.2408542087 & -0.0083976373 & \mathrm{C} \\ \mathrm{C} & 2.8883858968 & 0.0004391084 & -0.0052388730 & \mathrm{O} \\ \mathrm{C} & 3.6011188942 & -1.2401722511 & 0.0000861830 & \mathrm{O} \\ \mathrm{C} & 2.8601843736 & 2.4231589443 & -0.0141386759 & \mathrm{O} \\ \mathrm{C} & 1.4708480955 & 2.4225434223 & -0.0164022650 & \mathrm{O} \\ \mathrm{C} & 0.7321405245 & 1.2370963867 & -0.0131433828 & \mathrm{H} \\ \mathrm{C} & 1.4499908973 & 0.0004939399 & -0.0079197925 & \mathrm{H} \\ \mathrm{C} & 0.7322911652 & -1.2361622764 & -0.0057999105 & \mathrm{H} \\ \mathrm{C} & 1.4706555965 & -2.4217815571 & -0.0025580066 & \mathrm{H} \\ \mathrm{C} & 2.8598285327 & -2.4225197153 & 0.0004217768 & \mathrm{H} \\ \mathrm{C} & -0.7317674598 & 1.2369478141 & -0.0150889574 & \mathrm{H} \\ \mathrm{C} & -1.4493789781 & 0.0001975700 & -0.0119379818 & \mathrm{H} \\ \mathrm{C} & -0.7314366106 & -1.2363089428 & -0.0072254488 & \mathrm{H} \\ \mathrm{C} & -1.4707044712 & 2.4222487874 & -0.0195072006 & \mathrm{H} \\ \mathrm{C} & -2.8600417563 & 2.4225808431 & -0.0215542995 & \mathrm{H} \\ \mathrm{C} & -3.6012373281 & 1.2401204206 & -0.0190080019 & \mathrm{H} \\ \mathrm{C} & -2.8877760464 & -0.0001505098 & -0.0132895309 & \mathrm{H} \\ \mathrm{C} & -3.6002718736 & -1.2409029118 & -0.0088588590 & \mathrm{H} \\ \mathrm{C} & -2.8587413312 & -2.4230952957 & -0.0047780302 & \mathrm{H} \\ \mathrm{C} & -1.4695665038 & -2.4220731233 & -0.0038832242 & \mathrm{H} \\ \mathrm{C} & -5.0667684598 & 1.2452380524 & -0.0219447838 & \mathrm{H} \\ \mathrm{C} & -5.7673545149 & -0.0014225008 & -0.0158346709 & \mathrm{C} \\ \mathrm{C} & -5.0655473460 & -1.2471426602 & -0.0084684540 & \mathrm{H} \\ \mathrm{C} & -5.8147119832 & 2.4286870215 & -0.0303146139 & \mathrm{H} \\ \mathrm{C} & -7.2112590705 & 2.4193116262 & -0.0317555015 & \mathrm{H} \\ & & & & \end{array}$

$\begin{array}{ccc}-7.9084772968 & 1.2213808574 & -0.0249176605 \\ -7.1969992193 & -0.0022671731 & -0.0170251675 \\ -7.9081180942 & -1.2264376025 & -0.0102396663 \\ -7.2085697647 & -2.4233274074 & -0.0020733264 \\ -5.8122985331 & -2.4312628659 & -0.0011891561 \\ -9.3875542364 & 1.2371769782 & -0.0259892466 \\ -10.0355186360 & -0.0054971119 & -0.0195314960 \\ -9.3883051067 & -1.2522268444 & -0.0113949263 \\ 9.3891365405 & -1.2503624519 & 0.0172525901 \\ 10.0361163509 & -0.0034885768 & 0.0123289015 \\ 9.3879156316 & 1.2390572603 & 0.0047836902 \\ 10.0257395727 & -2.2969430305 & 0.0229566065 \\ 10.0423598340 & 2.2747350714 & 0.0003600702 \\ -10.0247589215 & -2.2989033826 & -0.0055326466 \\ -10.0421505050 & 2.2727444638 & -0.0320966210 \\ 7.7730737601 & 3.3492083492 & -0.0094664258 \\ 5.3167070449 & 3.3919163709 & -0.0150791499 \\ 5.3147588141 & -3.3919032155 & 0.0166803974 \\ 7.7701097216 & -3.3508229129 & 0.0232652690 \\ 3.3616355808 & 3.3836567551 & -0.0170312740 \\ 0.9698829624 & 3.3829721337 & -0.0210179240 \\ 0.9695969143 & -3.3821409781 & -0.0023960842 \\ 3.3612312271 & -3.3830155078 & 0.0028077976 \\ -0.9699243854 & 3.3827838128 & -0.0214209180 \\ -3.3616865495 & 3.3829779928 & -0.0249110525 \\ -3.3599463255 & -3.3836933028 & -0.0023317149 \\ -0.9683150193 & -3.3823242798 & -0.0000807901 \\ -5.3167136716 & 3.3908136012 & -0.0361398836 \\ -7.7730843002 & 3.3476055689 & -0.0382364433 \\ -7.7689118316 & -3.3523688268 & 0.0036497482 \\ -5.3135461615 & -3.3929553430 & 0.0057001766 \\ -11.5019754843 & 0.0243284891 & -0.0208750296 \\ -11.8523720708 & -1.0051383937 & -0.0177621236 \\ -11.8602572838 & 0.5516398458 & -0.9082987197 \\ -11.8620900329 & 0.5571024008 & 0.8626133766\end{array}$


$\begin{array}{llllll}\text { C } & 11.5025541840 & 0.0267497092 & 0.0149843240 & \mathrm{H}\end{array}$

H $\quad 11.8532495505 \quad-1.0025943190 \quad 0.0217562307 \quad \mathrm{H}$
11.8598829056 11.8634347812
0.5621996952

0.5515943342
0.8979379426 $-0.8729204908$

$N, N$-dimethyl QDI: Singlet Excited State

\begin{tabular}{|c|c|c|c|}
\hline $\mathrm{C}$ & 7.1901641996 & 2.4267726163 & 0.0001210557 \\
\hline $\mathrm{C}$ & 5.7999755838 & 2.4374651300 & -0.0002257954 \\
\hline $\mathrm{C}$ & 5.0466171280 & 1.2449627940 & -0.0002804110 \\
\hline $\mathrm{C}$ & 5.7483639625 & -0.0003677422 & -0.0001441663 \\
\hline $\mathrm{C}$ & 7.1786773161 & -0.0009057670 & 0.0003468641 \\
\hline $\mathrm{C}$ & 7.8894284754 & 1.2227754673 & 0.0004736784 \\
\hline $\mathrm{C}$ & 5.0458038466 & -1.2450433574 & -0.0004550636 \\
\hline $\mathrm{C}$ & 5.7984862641 & -2.4378544807 & 0.0000405043 \\
\hline $\mathrm{C}$ & 7.1884255922 & -2.4279834671 & 0.0005778766 \\
\hline $\mathrm{C}$ & 7.8896130173 & -1.2247535247 & 0.0006876672 \\
\hline $\mathrm{C}$ & 3.5944248806 & 1.2396717398 & -0.0003816876 \\
\hline $\mathrm{C}$ & 2.8803416219 & 0.0003911711 & -0.0008700201 \\
\hline $\mathrm{C}$ & 3.5938644147 & -1.2391321959 & -0.0012497312 \\
\hline $\mathrm{C}$ & 2.8465465240 & 2.4360539652 & -0.0000208131 \\
\hline $\mathrm{C}$ & 1.4696313095 & 2.4362814142 & -0.0000593916 \\
\hline $\mathrm{C}$ & 0.7227280982 & 1.2356076931 & -0.0004870568 \\
\hline $\mathrm{C}$ & 1.4418714857 & 0.0005387659 & -0.0010299212 \\
\hline $\mathrm{C}$ & 0.7226482178 & -1.2344586837 & -0.0017652642 \\
\hline $\mathrm{C}$ & 1.4691351818 & -2.4353476681 & -0.0027055603 \\
\hline $\mathrm{C}$ & 2.8459277907 & -2.4354128669 & -0.0024378364 \\
\hline $\mathrm{C}$ & -0.7227278549 & 1.2356074854 & -0.0003575569 \\
\hline $\mathrm{C}$ & -1.4418707631 & 0.0005385188 & -0.0008798256 \\
\hline $\mathrm{C}$ & -0.7226469219 & -1.2344589598 & -0.0015810052 \\
\hline $\mathrm{C}$ & -1.4696314642 & 2.4362806767 & 0.0004382733 \\
\hline $\mathrm{C}$ & -2.8465468394 & 2.4360532898 & 0.0004769799 \\
\hline $\mathrm{C}$ & -3.5944259293 & 1.2396710737 & -0.0002281126 \\
\hline $\mathrm{C}$ & -2.8803409538 & 0.0003908290 & -0.0006471031 \\
\hline $\mathrm{C}$ & -3.5938617838 & -1.2391334332 & -0.0008053331 \\
\hline $\mathrm{C}$ & -2.8459256318 & -2.4354143569 & -0.0016634174 \\
\hline $\mathrm{C}$ & -1.4691330841 & -2.4353487638 & -0.0020508604 \\
\hline $\mathrm{C}$ & -5.0466195617 & 1.2449592299 & -0.0004561341 \\
\hline $\mathrm{C}$ & -5.7483630402 & -0.0003737417 & -0.0000811902 \\
\hline $\mathrm{C}$ & -5.0457999760 & -1.2450474297 & -0.0000273668 \\
\hline $\mathrm{C}$ & -5.7999836408 & 2.4374585176 & -0.0009612256 \\
\hline $\mathrm{C}$ & -7.1901724156 & 2.4267598682 & -0.0007280974 \\
\hline $\mathrm{C}$ & -7.8894316890 & 1.2227605557 & -0.0000287065 \\
\hline $\mathrm{C}$ & -7.1786762349 & -0.0009167640 & 0.0002556554 \\
\hline
\end{tabular}

$N, N$-dimethyl QDI: Triplet State $\left(\mathrm{T}_{1}\right)$

\begin{abstract}
7.1781991310
2.4296602246

5.7919396174

2.4413570522

5.0336940581

1.2441187669

5.7358115550

7.1668558342

$-0.0003226212$

$-0.0009428769$

7.8776642286

1.2219843353

$5.0327901050 \quad-1.2440365370$

$5.7901724139-2.4416826821$

$7.1761865215 \quad-2.4309987984$

$7.8776773020-1.2241485068$

0.0008717713 0.0005596451

0.0006042064

0.0008660639

0.0014524947

0.0014698287

0.0004191486

0.0006646241

0.0012435221

0.0017742227
\end{abstract}

$-7.8896075632-1.2247668765$

$-7.1884167339-2.4279950111$

$-5.7984771976-2.4378610545$

$-9.3660463214 \quad 1.2390516536$

$-10.0140773696-0.0034460635$

$-9.3672695937-1.2503858792$

$9.3672785541-1.2503649509$

$10.0140826235 \quad-0.0034221753$

$9.3660447679 \quad 1.2390734896$

$10.0040360685-2.2986195069$

$10.0205537012 \quad 2.2764608910$

$-10.0040162367-2.2986436939$

$\begin{array}{ll}-10.0205598400 & 2.2764348005\end{array}$

$\begin{array}{ll}7.7550653287 & 3.3531584735\end{array}$

$\begin{array}{lll}5.3001802212 & 3.3985807641\end{array}$

$5.2983568360-3.3987693681$

$7.7522057495 \quad-3.3548995785$

3.3532721349

0.9642708343

3.3933875716

0.9635917387

3.3940539980

$-3.3929966317$

3.3524822624

$-3.3928193263$

$-0.9642708591$

$-3.3532711782$

3.3940529731

3.3933871268

$-3.3524811231-3.3928209638$

$-0.9635892195-3.3929979775$

$\begin{array}{ll}-5.3001936144 & 3.3985765266\end{array}$

$\begin{array}{ll}-7.7550783039 & 3.3531426304\end{array}$

$-7.7521937123$

$-3.3549134142$

$-5.2983429303$

$-11.4804263681$

$-3.3987737513$

$-11.8309041328$

0.0267517201

$-1.0027351146$

$-11.8396937806$

0.5583289867

0.5557925555

$-11.8394754436$

0.0267777648

11.4804317399

$-1.0027098101$

11.8309101350

0.5576326490

11.8397170181

0.5565428330
0.0008859625

0.0012140766

0.0007488082

0.0003835036

0.0008796430

0.0013166466

0.0012756017

0.0011870321

0.0008876950

0.0010885721

0.0007124000

0.0020286153

0.0003126985

0.0001475901

$-0.0005040690$

0.0001785610

0.0009760287

0.0003655571

0.0002126294

$-0.0037667312$

$-0.0033488731$

0.0011389468

0.0012859452

$-0.0021644051$

$-0.0026601481$

$-0.0016886313$

$-0.0010855679$

0.0018409644

0.0011504524

0.0012660899

$-0.0000188034$

$-0.8833290110$

0.8875123327

0.0013334038

0.0019802998

0.8864736344

$-0.8843692242$
C

C

C

C

$\mathrm{C}$

C

C

C

$\mathrm{C}$

C $\begin{array}{llll}3.5910421392 & 1.2383746367 & 0.0003085203\end{array}$

$\begin{array}{llll}2.8770098807 & 0.0005638715 & -0.0001511166\end{array}$

$\begin{array}{llll}3.5903741427 & -1.2375589845 & -0.0003640216\end{array}$

$\begin{array}{llll}2.8373109944 & 2.4438991343 & 0.0004251463\end{array}$

$\begin{array}{llll}1.4705632649 & 2.4444708502 & 0.0002375627\end{array}$

$\begin{array}{llll}0.7157442526 & 1.2342709742 & -0.0001312076\end{array}$

$\begin{array}{llll}1.4350316463 & 0.0007441154 & -0.0004615652\end{array}$

$\begin{array}{llll}0.7156674087 & -1.2327136698 & -0.0010975380\end{array}$

$\begin{array}{llll}1.4699661217 & -2.4431811796 & -0.0017658499\end{array}$

$\begin{array}{llll}2.8365809356 & -2.4429636905 & -0.0014072170\end{array}$ 


$\begin{array}{lcccc}\mathrm{C} & -0.7157431071 & 1.2342705493 & -0.0001511858 & \mathrm{O} \\ \mathrm{C} & -1.4350296275 & 0.0007433801 & -0.0006097902 & \mathrm{O} \\ \mathrm{C} & -0.7156644513 & -1.2327141073 & -0.0011094674 & \mathrm{O} \\ \mathrm{C} & -1.4705630380 & 2.4444696629 & 0.0004159401 & \mathrm{H} \\ \mathrm{C} & -2.8373109378 & 2.4438971715 & 0.0003665215 & \mathrm{H} \\ \mathrm{C} & -3.5910421398 & 1.2383724894 & -0.0002342809 & \mathrm{H} \\ \mathrm{C} & -2.8770078779 & 0.0005624719 & -0.0005462881 & \mathrm{H} \\ \mathrm{C} & -3.5903700548 & -1.2375612884 & -0.0007726698 & \mathrm{H} \\ \mathrm{C} & -2.8365769559 & -2.4429657281 & -0.0014190313 & \mathrm{H} \\ \mathrm{C} & -1.4699620976 & -2.4431823478 & -0.0016019814 & \mathrm{H} \\ \mathrm{C} & -5.0336954982 & 1.2441135666 & -0.0004562877 & \mathrm{H} \\ \mathrm{C} & -5.7358096318 & -0.0003299489 & -0.0003093909 & \mathrm{H} \\ \mathrm{C} & -5.0327848994 & -1.2440418767 & -0.0003166031 & \mathrm{H} \\ \mathrm{C} & -5.7919464622 & 2.4413487595 & -0.0007504529 & \mathrm{H} \\ \mathrm{C} & -7.1782062629 & 2.4296468671 & -0.0006226026 & \mathrm{H} \\ \mathrm{C} & -7.8776671795 & 1.2219686991 & -0.0002564967 & \mathrm{H} \\ \mathrm{C} & -7.1668537980 & -0.0009551562 & -0.0001268779 & \mathrm{H} \\ \mathrm{C} & -7.8776702224 & -1.2241632891 & 0.0002127648 & \mathrm{H} \\ \mathrm{C} & -7.1761759725 & -2.4310114060 & 0.0004061833 & \mathrm{H} \\ \mathrm{C} & -5.7901616799 & -2.4416907310 & 0.0001205877 & \mathrm{C} \\ \mathrm{C} & -9.3540621359 & 1.2381448305 & -0.0000064172 & \mathrm{H} \\ \mathrm{N} & -10.0027478071 & -0.0036454549 & 0.0003121786 & \mathrm{H} \\ \mathrm{C} & -9.3551578547 & -1.2497467404 & 0.0004816139 & \mathrm{H} \\ \mathrm{C} & 9.3551672159 & -1.2497257723 & 0.0028033920 & \mathrm{C} \\ \mathrm{N} & 10.0027494759 & -0.0036226847 & 0.0011659401 & \mathrm{H} \\ \mathrm{C} & 9.3540594526 & 1.2381665466 & 0.0016612705 & \mathrm{H} \\ \mathrm{O} & 9.9907503197 & -2.2984531358 & -0.0010260412 & \mathrm{H}\end{array}$

$\begin{array}{ccc}10.0075108462 & 2.2759931678 & -0.0010810985 \\ -9.9907310883 & -2.2984854916 & 0.0012105012 \\ -10.0075210036 & 2.2759694410 & 0.0003462441 \\ 7.7449745114 & 3.3547695233 & 0.0007528358 \\ 5.2916151575 & 3.4019518015 & 0.0001353283 \\ 5.2893833449 & -3.4020039350 & 0.0005810850 \\ 7.7417226560 & -3.3567126078 & 0.0015743257 \\ 3.3474527971 & 3.3993638426 & 0.0007374443 \\ 0.9623652364 & 3.4006402871 & 0.0003391250 \\ 0.9615478082 & -3.3991950123 & -0.0027059577 \\ 3.3465115007 & -3.3985117046 & -0.0021275196 \\ -0.9623655359 & 3.4006392429 & 0.0010182183 \\ -3.3474521068 & 3.3993617172 & 0.0010081244 \\ -3.3465081018 & -3.3985139703 & -0.0019191923 \\ -0.9615430985 & -3.3991961000 & -0.0021105479 \\ -5.2916267680 & 3.4019456277 & -0.0012153239 \\ -7.7449853612 & 3.3547538412 & -0.0008295318 \\ -7.7417091949 & -3.3567271918 & 0.0007804117 \\ -5.2893679455 & -3.4020098986 & 0.0003863623 \\ -11.4690760751 & 0.0264705753 & 0.0007863747 \\ -11.8195974062 & -1.0029668792 & -0.0006244729 \\ -11.8283343381 & 0.5579862897 & -0.8837616836 \\ -11.8280225612 & 0.5552021630 & 0.8871715553 \\ 11.4690754383 & 0.0264928364 & -0.0012979630 \\ 11.8195957877 & -1.0029466874 & -0.0009623370 \\ 11.8295747509 & 0.5573980305 & 0.8831248720 \\ 11.8267793202 & 0.5558352087 & -0.8878078207\end{array}$ 


\section{References}

1. Chen, M.; Bae, Y. J.; Mauck, C. M.; Mandal, A.; Young, R. M.; Wasielewski, M. R., Singlet fission in covalent terrylenediimide dimers: Probing the nature of the multiexciton state using femtosecond mid-infrared spectroscopy. J. Am. Chem. Soc. 2018, 140 (29), 9184-9192. 